\title{
The high level of RANTES in the ectopic milieu recruits macrophages and induces their tolerance in progression of endometriosis
}

\author{
Xiao-Qiu Wang ${ }^{1 \star}$, Jing Yu ${ }^{1 *}$, Xue-Zhen Luo', Ying-Li Shi ${ }^{1}$, Yun Wang ${ }^{1}$, Ling Wang ${ }^{1}$ \\ and Da-Jin $\mathrm{Li}^{1,2}$ \\ ${ }^{1}$ Laboratory for Reproductive Immunology, Hospital and Institute of Obstetrics and Gynecology, IBS, Fudan University Shanghai Medical College, Shanghai 200011, \\ People's Republic of China \\ ${ }^{2}$ Department of Obstetrics and Gynecology, Hainan Medical College Affiliated Hospital, Haikou 570102, People's Republic of China \\ (Correspondence should be addressed to D-J Li at Laboratory for Reproductive Immunology, Hospital and Institute of Obstetrics and Gynecology, IBS, \\ Fudan University Shanghai Medical College; Email: dji@ @shmu.edu.cn) \\ *(X-Q Wang and $\mathrm{J}$ Yu contributed equally to this work $)$
}

\begin{abstract}
RANTES ( $C-C$ chemokine, regulated on activation, normal $T$ cell expressed and secreted) is involved in progression of endometriosis, but the precise mechanism is understood inadequately. This study is to elucidate the roles of RANTES in macrophage recruitment and tolerance in the endometriotic milieu. The expression of RANTES was analyzed by immunohistochemistry. The cell co-cultures were applied to simulate the endometriotic milieu to investigate the regulation of RANTES secretion and its receptor CCR1 expression. Transwell migration assay was used for chemotaxis of U937 cells (macrophage line) to endometrial stromal cells (ESCs) and/or human pelvic mesothelial cells. The expression of CCR1 was analyzed by RT-PCR and qPCR in transcription and by western blot in translation respectively. Concentrations of RANTES, IL10, and IL12p70 were determined by ELISA. The phenotype of U937 cells and apoptosis of ESCs were analyzed by flow cytometry. We have found that the expression of RANTES is significantly higher in the endometriotic tissue and eutopic endometrium than that of the normal endometrium without endometriosis. The combination of $17 \beta$-estradiol and dioxin 2,3,7,8-tetrachlorodibenzo-p-dioxin increases significantly RANTES secretion in the endometriosis-associated cell co-culture which can recruit more macrophages, upregulate CCR1 expression, and induce tolerant phenotype, which inhibits the apoptosis of ESC in the milieu. In conclusion, the higher levels of RANTES in the ectopic milieu facilitate the onset and progression of endometriosis by macrophage recruitment and tolerance that in turn inhibits apoptosis and enhances growth of ESC.
\end{abstract}

Journal of Molecular Endocrinology (2010) 45, 291-299

\section{Introduction}

Endometriosis is a common gynecological disorder in fertile women that is characterized by the presence and growth of functional endometrial tissues outside the uterine cavity. Although it has been generally accepted that implantation of endometrial cells and fragments refluxed during the menstrual period is the precondition for the onset of endometriosis, we are still perplexed why the endometrium cannot be efficiently cleared in the ectopic milieu. The number of leukocytes, particularly macrophages, increases in the peritoneal fluid of patients with endometriosis (Hill et al. 1988). These macrophages seem to have phenotypic and functional alterations leading to poor phagocytotic capacity, which is closely related to the severity of endometriosis (Raiter-Tenenbaum et al. 1998). However, the mechanisms by which the ectopic milieu modulates macrophage functions, especially tolerance, have not been fully elucidated. Our previous work has indicated that macrophages are involved in ectopic adhesion, implantation, and growth of the endometriotic tissue instead of clearing (Shi et al. 2006, 2007, $\mathrm{Yu}$ et al. 2008). Therefore, the peritoneal macrophages may contribute to the development of endometriosis. An understanding of the regulatory mechanism that controls macrophage functions is valuable to prophylaxis and therapeutics for endometriosis.

RANTES, one of the members of $\mathrm{C}-\mathrm{C}$ chemokine family, is found to be elevated in peritoneal fluid of women with endometriosis, and commensurates with the stage of the disease which appears to mediate chemotactic activity of the monocytes in peritoneal cavity (Hornung et al. 2001a,b), suggesting that it might contribute to the progression of this disease. However, the precise mechanism underlying roles of RANTES in endometriosis, has not been well elucidated. It has been known that RANTES mRNA and protein expression can be induced by the pro-inflammatory cytokines IL1 $\beta$, tumor necrosis factor- $\alpha(\mathrm{TNF}-\alpha)$, and interferon $\gamma$ 
(IFN- $\gamma$ ) in the endometrial stroma (Hornung et al. 1997, Lebovic et al. 2001). Treatment of the endometrial cells with estradiol before stimulation with IL1 $\beta$ results in an increase in RANTES transcription and secretion (Akoum et al. 2002).

Environmental contaminant is also involved in the pathogenesis of endometriosis. Exposure to 2,3,7,8tetrachlorodibenzo-p-dioxin (TCDD), a highly toxic environmental contaminant, is associated with an increased prevalence and severity of endometriosis (Rier \& Foster 2002). TCDD appears to be antagonistic and analogous to estrogen, so effects of TCDD on the action of estrogen and inflammation may be mainly mechanic in the pathogenesis of endometriosis. Our previous work has shown that the combination of $17 \beta$-estradiol $\left(\mathrm{E}_{2}\right)$ and TCDD upregulates CXCR1 and CCR8 expressions in endometrial stromal cells (ESCs), and promotes the secretion of their respective ligands, IL8 and I-309, in co-culture of the endometriotic focusassociated cells (Shi et al. 2006, 2007). We have also found that the combination of $\mathrm{E}_{2}$ and TCDD increases secretion of RANTES in the co-culture that increases the invasion of ESCs (Yu et al. 2008).

Macrophages have been classified along what is viewed as a linear scale: M1 designation is reserved for classically activated macrophages, and the M2 for tolerant macrophages (Martinez et al. 2008). M1 macrophages that are induced by the combination of IFN- $\gamma$ and TNF can produce high levels of proinflammatory cytokines and mediators as important components of host defense (O'Shea \& Murray 2008). In addition to immune complexes, other factors can provide a signal for the differentiation of M2 macrophages, including apoptotic cells (Erwig \& Henson 2007), adenosine (Hasko et al. 2007), and hyaluronan of moderate weight (Kuang et al. 2007). M2 macrophages that produce high levels of the immunosuppressive cytokine IL10 may dampen the immune response and limit inflammation (Mosser 2003). Some tumor-associated macrophages may share characteristics with M2 macrophages (Biswas et al. 2006). In addition to IL10 production, M2 macrophages also downregulate IL12 production, and then the ratio of IL10 to IL12 can be used to define M2 macrophage (Martinez et al. 2008). Because IL10 can inhibit the production and activity of many kinds of pro-inflammatory cytokines, M2 macrophages are potent inhibitors of inflammation, despite the fact that they retain the ability to produce many proinflammatory cytokines. M1 macrophages have low expression of CD14 and high expression of HLA-DR and CD86 on their surface, while M2 macrophages have increased expression of $\mathrm{CD} 14$ and decreased expression of HLA-DR and CD86, so the altered phenotype can be also applied for identification of M2 macrophages (Kuang et al. 2007).
In this study, we first observed the effects of $\mathrm{E}_{2}$ and TCDD on recruiting macrophages mediated by RANTES, and then analyzed the expression of CCR1 on macrophages. To better understand the role of RANTES in the progression of endometriosis, we investigated the secretion of IL10 and IL12p70 and the expression of CD14, HLA-DR, and CD86 in macrophages for the regulation of RANTES in the tolerance formation of macrophage. Lastly, we investigated the effect of the tolerant macrophages on apoptosis of ESC.

\section{Materials and methods}

\section{Tissue collection and cell culture}

All the normal endometrium, eutopic endometrium, and endometriotic tissues were obtained from 20 patients with or without endometriosis (mean age 42.5 years; range $34-46$ ), which had been confirmed by laparoscopy in the Hospital of Obstetrics and Gynecology, Fudan University Shanghai Medical College. The endometriosis patients were classified according to the revised American Fertility Society (AFS) classification: six in Stage 2 and nine in Stage 3. The normal endometrium was from five patients undergoing surgery for CIN II-III. These patients had not received any GnRH analog or other hormonal drug in the 6 months prior to surgery, and the informed consent was obtained before surgery from each patient using protocols approved by the Human Investigation Committee in the Hospital of Obstetrics and Gynecology, Fudan University Shanghai Medical College. All the samples were obtained in the proliferative phase of the menstrual cycle, which was confirmed histologically according to the established criteria. The minced eutopic endometrium was digested with collagenase type I ( $0 \cdot 1 \%$; Sigma) for $30 \mathrm{~min}$ at $37^{\circ} \mathrm{C}$ with constant agitation. The tissue pieces were filtered through a 200 and $400 \mu \mathrm{m}$ wire sieve to remove debris and epithelial cells. Following gentle centrifugation, the supernatant was discarded, and the cells were resuspended in DMEM/F-12 (Gibco). The cell suspension was layered over Ficoll, and centrifuged at $800 \mathrm{~g}$ for $20 \mathrm{~min}$ to further remove leukocytes and erythrocytes, and the middle layer was collected and then washed with D-Hanks. The ESCs were cultured in a flask, and allowed to adhere for $20 \mathrm{~min}$. The adherent stromal cells were cultured as monolayer with DMEM/F-12 containing 10\% FCS (Hyclone, Logan, UT, USA), $20 \mathrm{mmol} / \mathrm{l}$ HEPES, $100 \mathrm{IU} / \mathrm{ml}$ penicillin, and $100 \mathrm{mg} / \mathrm{ml}$ streptomycin and incubated in $5 \% \mathrm{CO}_{2}$ at $37^{\circ} \mathrm{C}$. The purity of ESCs is over $95 \%$.

HMrSV5 (HPMC, a human peritoneal mesothelial cell line provided by Prof. Jian Yao, the First People's 
Hospital, Shanghai, China) and human monocyte U937 cell line (purchased from Bank of Cell, Chinese Academy of Sciences, Shanghai, China) were maintained in DMEM (Gibco) with 10\% FCS and RPMI 1640 medium (Life Technologies) with $10 \%$ bovine calf serum respectively and containing $20 \mathrm{mmol} / \mathrm{l}$ HEPES, $100 \mathrm{IU} / \mathrm{ml}$ penicillin, and $100 \mathrm{mg} / \mathrm{ml}$ streptomycin at $37^{\circ} \mathrm{C}$ in a humidified, $5 \% \mathrm{CO}_{2}$ incubator. The medium was changed every other day.

\section{Immunohistochemistry}

The normal endometrium, eutopic endometrial, and endometriotic tissues were cut into serial sections of $5 \mu \mathrm{m}$ and were stained by Vectastain Elite ABC kit (Vector Laboratories, Burlingame, CA, USA). An immunoperoxidase staining was performed by monoclonal mouse antihuman RANTES antibody $(15 \mu \mathrm{g} / \mathrm{ml}$; R\&D Systems, Wiesbaden, Germany).

\section{Cell co-culture unit}

\section{Contact co-culture of two sorts of cells}

The ESCs or HPMCs were cultured in 24-well plates at a concentration of $1 \times 10^{5}$ cells/well until adhering to the flask wall. The medium was removed, and then ESCs or U937 cells were placed respectively over HPMC or ESC respectively at the same concentration. The cells were cultured in a final volume of $1 \mathrm{ml}$ fresh DMEM with $2.5 \%$ FCS for $48 \mathrm{~h}$. HPMCs, ESCs, and U937 cells in $1 \times 10^{5}$ cells/well were cultured alone as controls. Each experiment was carried out in triplicate and repeated three times.

\section{Contact co-culture of U937-ESC-HPMC}

The three sorts of cells were plated in the proportion of 1:1:1. HPMCs were cultured in 24-well plates at a concentration of $1 \times 10^{5}$ cells/well until adhering to the plastic bottom. The medium was removed, and then ESCs were applied over HPMCs at the same concentration. After ESCs adhered to the plastic bottom and HPMCs, the medium was removed again. U937 cells were applied over ESCs and HPMCs at the same concentration. The cells were cultured in a final volume of $1 \mathrm{ml}$ fresh DMEM with 2.5\% FCS for $48 \mathrm{~h}$. Each experiment was carried out in triplicate and repeated three times.

\section{Noncontact transwell co-culture of two sorts of cells}

The two sorts of cells were also plated in the proportion of $1: 1$. The ESCs, HPMCs, and U937 cells at $1 \times 10^{5}$ cells/well were plated respectively at lower or upper compartment of Costar transwell cell culture chamber inserts $(0.4 \mu \mathrm{m}, 12 \mathrm{~mm}$ diameter $)$. Therefore, three different transwell co-cultures of two cells were established: E-H (ESC-HPMC), E-U (ESC-U937), and H-U (HPMC-U937) co-cultures. Each experiment was carried out in triplicate and repeated three times.

\section{Treatment in vitro with estrogen and/or TCDD}

After serum starvation for $12 \mathrm{~h}, \mathrm{U} 937$ cells were treated with $\mathrm{E}_{2}$ at a concentration ranging from $10^{-10}$ to $10^{-7} \mathrm{~mol} / \mathrm{l}$ (Sigma), TCDD at a concentration ranging from 0.01 to $10 \mathrm{nmol} / 1$ (Sigma), or the combination of $\mathrm{E}_{2}\left(10^{-8} \mathrm{~mol} / \mathrm{l}\right)$ and TCDD $(1 \mathrm{nmol} / \mathrm{l})$ for $48 \mathrm{~h}$. Every co-culture unit was treated with $\mathrm{E}_{2}\left(10^{-8} \mathrm{~mol} / \mathrm{l}\right)$ or TCDD $(1 \mathrm{nmol} / \mathrm{l})$ or with the combination of both for $48 \mathrm{~h}$, with vehicle (DMSO) as controls. Each experiment was carried out in triplicate and repeated three times.

\section{Transwell migration assay}

According to the different cells in the lower chamber, the following two groups were included: first group ESCs, HPMCs, or ESC-HPMC co-culture were put into the lower chamber, and U937 cells $\left(2 \times 10^{5}\right.$ cells/well $)$ were put into the upper chamber after incubation for $48 \mathrm{~h}$. Anti-RANTES-neutralizing antibody (R\&D Systems) at $2.5 \mu \mathrm{g} / \mathrm{ml}$ was added to several lower chambers half an hour before chemotaxis assay. After $3 \mathrm{~h}$ of incubation, the U937 cells in the lower chamber were counted. Second group - ESCs at $1 \times 10^{5}$ cells/well in the lower chamber are treated with $\mathrm{E}_{2}$ at $10^{-8} \mathrm{~mol} / \mathrm{l}$, TCDD at $1 \mathrm{nmol} / \mathrm{l}$, or the combination of both, and other experimental procedure is same as above. U937 cells were pretreated with anti-CCR1-neutralizing antibody (MBL, Nagoya, Japan) at $10 \mathrm{mg} / \mathrm{ml}$ or antiCCR5-neutralizing antibody (BD, Franklin Lakes, NJ, USA) at $5 \mathrm{mg} / \mathrm{ml}$ half an hour before chemotaxis assay for role of the receptors in U937 recruitment.

\section{Total RNA extraction and RT-PCR}

Total RNA of U937 cells was isolated by using Trizol reagent (Gibco). The RT reaction was performed by a nonamer primer and $1 \mu \mathrm{g}$ of RNA in a volume of $20 \mu \mathrm{l}$. PCR primers of CCR1 and the housekeeping gene glyceraldehyde-3-phosphate dehydrogenase (GAPDH) were as indicated elsewhere (Shi et al. 2006). The PCR cycles and conditions for denaturation, annealing, and elongation were 35 cycles, $1 \mathrm{~min}$ at $94^{\circ} \mathrm{C}, 30 \mathrm{~s}$ at $55^{\circ} \mathrm{C}$, and $30 \mathrm{~s}$ at $72{ }^{\circ} \mathrm{C}$ respectively. The PCR products were resolved in $1.5 \%$ agarose gel and visualized by ethidium bromide staining. 


\section{Quantitative real-time PCR}

Total RNA of U937 cells was isolated by using Trizol reagent (Gibco). Triplicate samples containing cDNA were prepared as mentioned above. SYBR Premix Ex Taq, ROX Reference Dye II (TaKaRa Biotechnology Co. Ltd, Kyoto, Japan), and specific primers were mixed and analyzed on an ABI7000 thermal cycler (Applied Biosystems, Foster City, CA, USA). The primer sequences of CCR1 and housekeeping gene GAPDH are indicated in Table 1 and were synthesized by Sangon Biotech Co., Ltd (Shanghai, China). The cycling conditions consisted of a denaturation step at $95^{\circ} \mathrm{C}$ for $30 \mathrm{~s}, 40$ cycles at $95^{\circ} \mathrm{C}$ for $5 \mathrm{~s}$, and a $34 \mathrm{~s}$ annealing step at $60{ }^{\circ} \mathrm{C}$. To determine the amount of gene product present in the sample, cycle time $\left(C_{\mathrm{t}}\right)$ was determined. The average $C_{\mathrm{t}}$ value was calculated from triplicate wells for each sample with each primer set. Most duplicate samples varied by $<0.5 C_{\mathrm{t}}$. The relative gene expression was determined by calculating $\Delta C_{\mathrm{t}}$ values $\left(\Delta C_{\mathrm{t}}\right)$ by subtracting the $C_{\mathrm{t}}$ value for $G A P D H$ primers from the $C_{\mathrm{t}}$ value for target gene primers. The relative fold expression for each gene was determined by comparing with controls in the experiment. The experiments were carried out in triplicate.

\section{Western blots}

Western blot analysis was performed by antihuman CCR1 polyclonal antibody (Abcam, Cambridge, UK), and was resolved on SDS-PAGE and transferred to immobilon PVDF membranes. After being soaked in blocking buffer, the membrane was incubated with primary antibody overnight at $4{ }^{\circ} \mathrm{C}$. The blots were developed using the HRP-linked secondary antibody and a chemiluminescent detection system. The experiments were repeated three times.

\section{ELISA for determination of RANTES, IL10, and IL12p70}

In ESC, HPMC, and U937, cultured alone and different two cells co-culture models, the culture supernatant was collected, and the concentration of RANTES was quantified by the ELISA kits (R\&D Systems). U937 cells $\left(1 \times 10^{6}\right.$ cells/well in 96 -well plates $)$ were cultured with or without the presence of recombinant human RANTES (rhRANTES; Peprotech, Rocky Hill, NJ, USA) at a concentration of $10 \mathrm{ng} / \mathrm{ml}$ for $0-96 \mathrm{~h}$, and the concentration of IL12p70 and IL10 in the culture supernatant was quantified by ELISA kits (R\&D Systems) according to the manufacturer's instructions. The limit of detection was $<2 \cdot 0 \mathrm{pg} / \mathrm{ml}$. Each experiment was carried out in triplicate, and repeated three times.

\section{Flow cytometry for phenotypes of macrophage}

The U937 cells $\left(5 \times 10^{5}\right.$ cells/well) were cultured in sixwell plates with or without the presence of rhRANTES (Peprotech) at a concentration of $10 \mathrm{ng} / \mathrm{ml}$ for $72 \mathrm{~h}$, after which, lipopolysaccharide (LPS; $10 \mathrm{ng} / \mathrm{ml}$ ) was added to several wells above for $24 \mathrm{~h}$, and then the cells were incubated for $30 \mathrm{~min}$ at room temperature with $80 \mu \mathrm{l}$ PBS containing $0 \cdot 2 \%$ BSA (PBS-BSA) supplemented with $20 \mu$ antihuman CD14, CD86, and HLADR antibody (eBioscience, San Diego, CA, USA). After that the cells were washed with PBS-BSA, and were analyzed by a FACScan flow cytometer (Becton Dickinson, Mountain View, CA, USA).

\section{Apoptosis assay for ESC}

To evaluate the early apoptosis, exposure of phosphatidylserine on a cell surface was examined. The ESCs were cultured in six-well plates with the concentration of $5 \times 10^{5}$ cells / well until adhering to the flask wall. The medium was removed, and then the U937 cells cultured alone or pretreated with $10 \mathrm{ng} / \mathrm{ml}$ rhRANTES for $72 \mathrm{~h}$ were placed over ESCs at the same concentration. After $48 \mathrm{~h}$, the ESCs were detached, washed, and resuspended to $1 \times 10^{5}$ cells in $100 \mu \mathrm{l}$ PBS containing $0 \cdot 2 \%$ BSA (PBS-BSA). The ESCs were then incubated for 15 min at room temperature with $5 \mu$ annexin V-FITC and $10 \mu \mathrm{l}$ propidium iodide (PI; Invitrogen). Here, the apoptosis cells are annexin V-FITC positive and PI negative.

\section{Statistical analysis}

All values are shown as mean \pm s.D., and are analyzed by variance (one-way ANOVA: post hoc analysis). P value $<0.05$ is considered statistically significant.

Table 1 Primer sequences of CCR1 and glyceraldehyde-3-phosphate dehydrogenase (GAPDH)

Size $(b p) \quad$ Primer sequences

Gene names CCR1 $G A P D H$
123

235
Sense: 5'-AAGGGCTTGGACTCAAGCAAGA-3' Antisense: 5'-TGGAGCCCACAGTCACCACTAC-3' Sense: 5'-GGGGAGCCAAAAGGGTCATCATCT-3' Antisense: $5^{\prime}$-GAGGGGCCATCCACAGTCTTCT-3' 


\section{Results}

Immunohistochemistry of RANTES in the normal endometrium, eutopic endometrium, and endometriotic tissues

Immunohistochemistry was used to localize RANTES protein in the frozen section. In the normal endometrium without endometriosis, stromal RANTES expression was observed in seven of the ten proliferative samples, and was hardly observed in the epithelial cells. In all of the 13 eutopic endometrial samples, we observed a significant increase in RANTES expression in epithelial and stromal cells; here the stromal cells were stained more intensely than the epithelial cells. The immunostain was localized in the cytoplasm and the membrane of epithelial and stromal cells. In 9 of the 11 endometriotic tissues, RANTES immunoreactivity in the stromal compartment was observed, but the epithelial cells were hardly observed. The optical density of RANTES immunostaining in the stromal compartment of the eutopic endometrial samples and endometriotic tissues appears significantly more intense than that of the normal endometrium without endometriosis $(P<0 \cdot 05$; Fig. 1).

\section{Effect of the co-culture and estradiol or/and TCDD on the secretion of RANTES}

The cultured U937 cells secreted more RANTES than ESC and HPMC did. The noncontact co-cultures by transwell had no effect on RANTES secretion. In the contact co-cultures of 24-well plates, neither U937 cells nor HPMCs co-cultured with ESCs affected RANTES secretion, whereas the co-culture of HPMC with U937 cells promoted significantly RANTES production and release $(P<0 \cdot 05$; Fig. $2 \mathrm{~A})$.

Either estradiol or TCDD tended to stimulate RANTES secretion in the contact HPMC-U937 co-culture, but showed no significant difference $(P>0 \cdot 05)$. The combination of them appeared to have a synergistic stimulatory effect on RANTES secretion in this co-culture $(P<0 \cdot 05$; Fig. $2 \mathrm{~B})$.
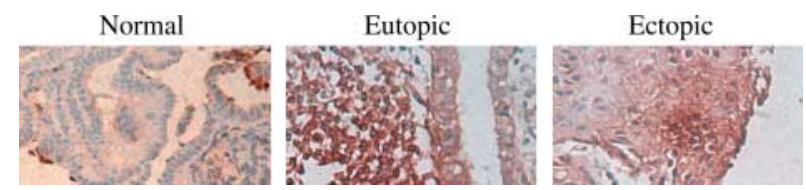

Figure 1 Immunohistochemistry of RANTES in the normal endometrium, eutopic endometrium, and the ectopic tissues.

Representative micrographs of immunohistochemical staining for RANTES in the normal endometrium, eutopic endometrium, and the endometriotic tissues. Magnification, $\times 400$.
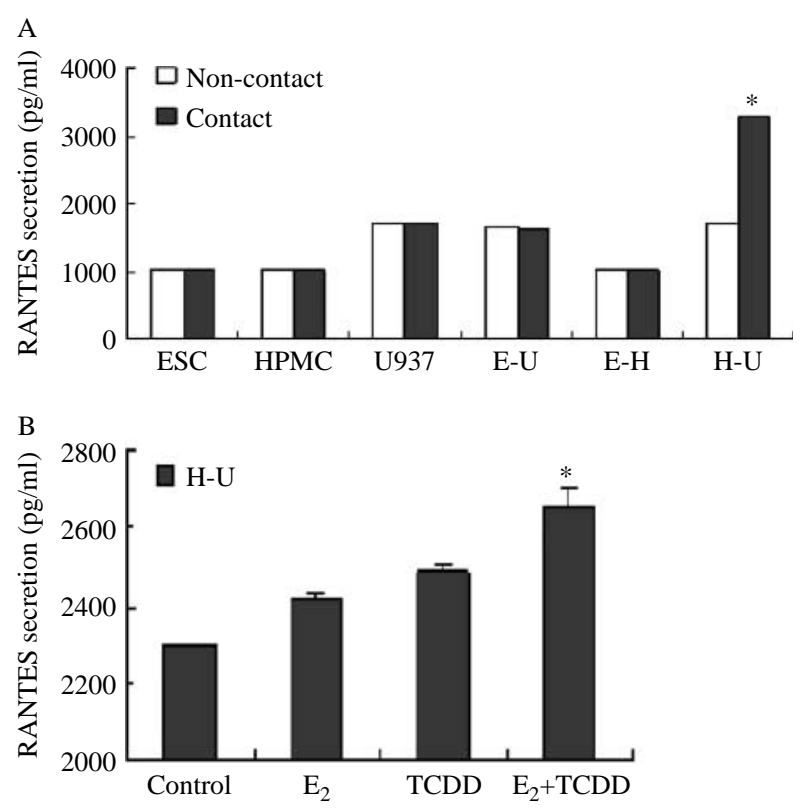

Figure 2 The $\mathrm{H}-\mathrm{U}$ co-culture and the combination of $17 \beta$-estradiol and TCDD promote the secretion of RANTES. ESCs, HPMCs, and $\mathrm{U} 937$ cells alone and $\mathrm{E}-\mathrm{U}, \mathrm{E}-\mathrm{H}$, and $\mathrm{H}-\mathrm{U}$ co-culture were cultured in the noncontact transwell or cell-cell contact 24-well plates for $48 \mathrm{~h}(\mathrm{~A})$. The $\mathrm{H}-\mathrm{U}$ co-culture in 24-well plates was treated with $17 \beta$-estradiol $\left(10^{-8} \mathrm{~mol} / \mathrm{l}\right)$ or/and TCDD $(1 \mathrm{nmol} / \mathrm{l})$ for $48 \mathrm{~h}(\mathrm{~B})$. The levels of RANTES in the supernatants were determined by ELISA. Data are expressed as mean \pm S.E.M. ${ }^{\star} P<0.05$ compared with others.

\section{Effect of the co-culture on U937 migration}

The ESC-HPMC co-culture could significantly enhance the chemotaxis of U937 compared with the control or ESCs or with HPMCs cultured alone, and anti-RANTES-neutralizing antibody partly inhibited this effect but this was still higher than that of ESCs or HPMCs cultured alone $(P<0 \cdot 01$; Fig. 3A).

\section{Effect of estradiol and/or TCDD on U937 migration in the co-culture}

$\mathrm{E}_{2}$ or TCDD alone had no effect on the migration of U937 cells to ESCs, but the combination of both could obviously promote the chemotaxis of U937 cells $(P<0 \cdot 01)$. Anti-CCR1, -CCR5, and-RANTES-neutralizing antibody inhibited partly the effect of the combination $(P<0 \cdot 01$; Fig. 3B $)$.

\section{Effect of estradiol or/and TCDD on CCR1 expression in U937 cells}

It was shown in Fig. 4 that the mRNA transcription and protein translation of CCR1 in U937 cells were increased in a dose-dependent manner after treated with $\mathrm{E}_{2}$ or TCDD. The combination of both could further promote the expression of CCR1 $(P<0 \cdot 01)$. 

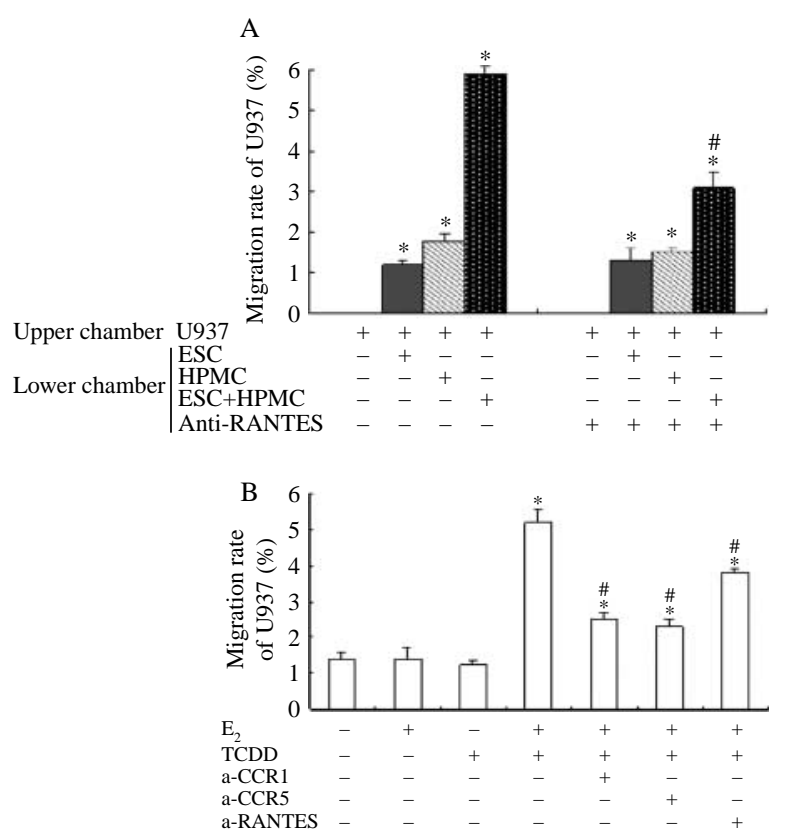

Figure 3 The co-culture and combination of $17 \beta$-estradiol and TCDD increase chemotaxis of U937 through secreting RANTES. In the transwell assay, ESCs, HPMCs, or ESC-HPMC co-culture were put in the lower chamber, and U937 cells were put in the upper chamber. The ESC-HPMC co-culture significantly increased the chemotaxis of U937, and anti-RANTES-neutralizing antibody partly inhibited the increased chemotaxis of U937 cells (A). The combination of $17 \beta$-estradiol and TCDD promoted migration of U937 cells to ESCs, and anti-CCR1, -CCR5, or -RANTES-neutralizing antibody partly inhibited the effect of $17 \beta$-estradiol and TCDD (B). ${ }^{*} P<0.01$ compared with control. ${ }^{\#} P<0.01$ compared with U-E-H co-culture unit control $(A)$ or ESC treated with the combination of $17 \beta$-estradiol and TCDD (B).

\section{Effects of the co-culture and estradiol or/and TCDD on the expression of CCR1 in U937 cells}

Co-culture could promote the expression of CCR1 in U937 cells compared to U937 cultured alone $(P<0 \cdot 01$; Fig. 5), especially the contact U-H-E co-culture. TCDD could promote the expression of CCR1 in U937 cells compared to the control in U-H and U-H-E co-culture $(P<0 \cdot 01$; Fig. 5$)$, and the combination of $\mathrm{E}_{2}$ with TCDD further increased the protein translation of CCR1 $(P<0 \cdot 01)$. In all the co-cultures, the combination of $\mathrm{E}_{2}$ with TCDD could upregulate the expression of CCR1 compared to the control $(P<0 \cdot 01$; Fig. 5$)$.

\section{RANTES can induce macrophage tolerance}

To investigate the effect of RANTES on polarization of macrophages, rhRANTES $(10 \mathrm{ng} / \mathrm{ml})$ was used to treat macrophages for $0-96 \mathrm{~h}$. The results revealed that RANTES could change the balance between the release of IL10 and IL12p70 of macrophages with the upregulation of IL10 production and downregulation of IL12p70 production (Fig. 6A). rhRANTES could induce macrophage tolerance with increased expression of CD14, and decreased expression of HLA-DR and CD86 $(P<0 \cdot 05$; Fig. 6B). The RANTES-treated cells were refractory to stimulation with LPS (Fig. 6B). These results above indicate that RANTES can induce the formation of tolerant phenotype of macrophages.

\section{The effect of tolerant macrophages on the apoptosis of ESCs}

ESCs co-cultured with U937 cells showed significantly lower apoptosis rate than ESCs cultured alone, and the apoptosis of ESCs did decrease further if co-cultured with the U937 cells pretreated with RANTES $(P<0 \cdot 05$; Fig. 7$)$.

\section{Discussion}

Endometriosis is a chronic inflammation disease. There are increased numbers of activated peritoneal macrophages in patients with endometriosis (Wu et al. 2002). These macrophages cannot effectively clear the ectopic endometrial cells (Raiter-Tenenbaum et al. 1998), and inversely secrete multiple kinds of inflammatory mediator to contribute to the progression of endometriosis, for instance IL8 and I-309 (Shi et al. 2006, 2007). In view of this, macrophages play an important role in the onset and development of this disease. However, our understanding of the macrophages' actions in the endometriotic milieu is still inadequate.
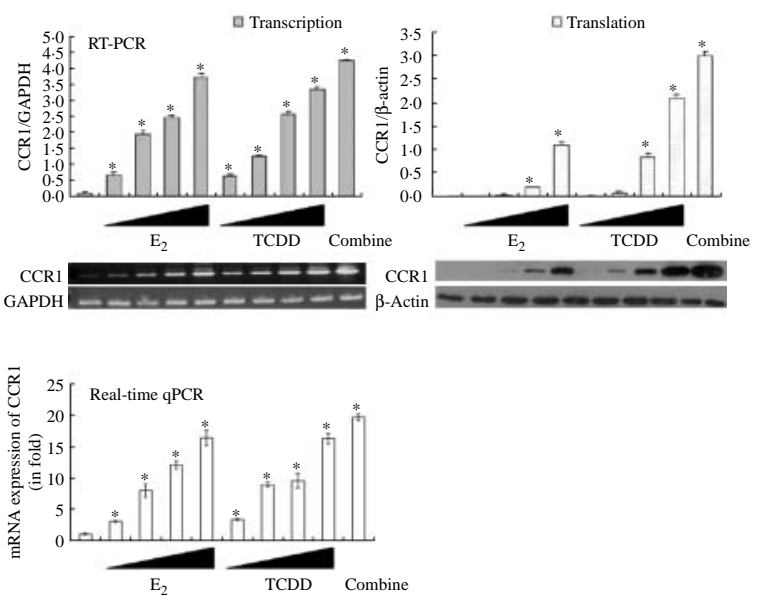

Figure 4 17 $\beta$-estradiol or/and TCDD promote the expression of CCR1 in U937. Treatment with $17 \beta$-estradiol $\left(10^{-10}, 10^{-9}, 10^{-8}\right.$, and $10^{-7} \mathrm{~mol} / \mathrm{l}$ successively) or TCDD $(0 \cdot 01,0 \cdot 1,1$, and $10 \mathrm{nmol} / \mathrm{l}$ successively) upregulated the mRNA transcription and protein translation of CCR1 in U937 cells; here, $17 \beta$-estradiol at $10^{-8} \mathrm{~mol} / / \mathrm{l}$ and TCDD at $1 \mathrm{nmol} / \mathrm{l}$ showed a synergetic effect. ${ }^{*} P<0.01$ compared with control. 


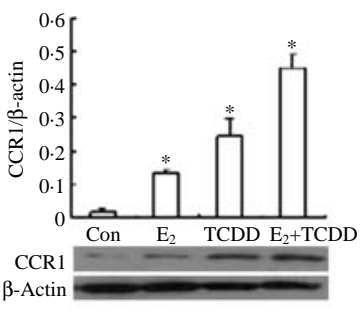

U937
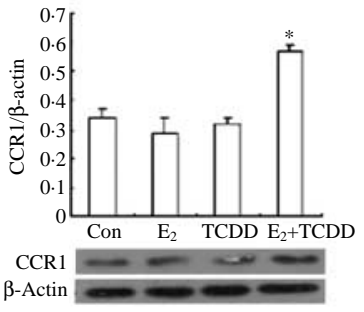

U937-ESC

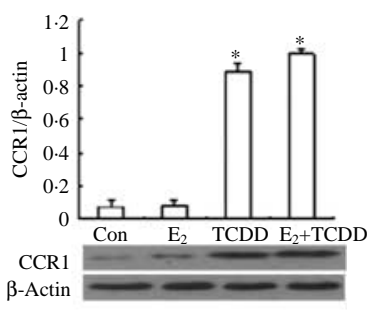

U937-HPMC

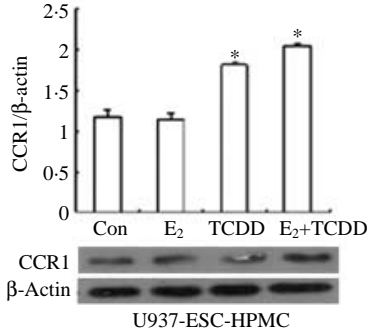

U937-ESC-HPMC
Figure 5 The combination of $17 \beta$-estradiol and TCDD upregulates CCR1 protein translation in U937 and the co-cultures. U937 cells were cultured alone or co-cultured with HPMCs, ESCs, or HPMCs and ESCs respectively, and were treated with $17 \beta$-estradiol at $10^{-8} \mathrm{~mol} / \mathrm{l}$ or/and TCDD at $1 \mathrm{nmol} / \mathrm{l}$ for $48 \mathrm{~h}$. The CCR 1 expression was detected by western blot. Data are expressed as mean \pm s.D. ${ }^{\star} P<0.01$ compared with control.

In this study, immunohistochemistry has confirmed that RANTES is translated significantly higher in the eutopic endometrium and ectopic tissues than in the normal endometrium. The observations are consistent with the findings of previous studies (Hornung et al. 2001a,b, Fang et al. 2009), which led us to propose that RANTES overexpression in the eutopic endometrium and endometriotic tissues may exert a potential role in the pathogenesis of endometriosis.

Our present data show that U937 cells spontaneously secrete much more RANTES than ESCs and HPMCs do. HPMCs cultured alone in nature secrete few RANTES, even if stimulated by the combination of $\mathrm{E}_{2}$ and TCDD (Wang XQ, unpublished observations). The direct cell contact of HPMC and U937 significantly promotes the secretion of RANTES, which means that the interaction of the endometriotic focus-associated cells does aggravate peritoneal inflammation by stimulating the release of RANTES. Further study showed that $\mathrm{E}_{2}$ or TCDD increased RANTES secretion in the HPMC-U937 co-culture unit, and the combination of them has a synergistic effect, which implies that the increased macrophages in peritoneal cavity of endometriosis interact with HPMCs to stimulate RANTES production. The chronic exposure to $\mathrm{E}_{2}$ and TCDD aggravates the inflammatory status by stimulating pro-inflammatory cytokine RANTES secretion, which leads to a persistent and serious inflammation, and finally results in the formation of an endometriotic focus.

The macrophages in peritoneal fluid are terminal cells without proliferation. Therefore, the monocytes' recruitment from peripheral blood into peritoneal fluid is an important step for the onset of endometriosis. It has been shown that RANTES accounts for the majority of the monocyte chemotactic activity in the ectopic ESC-conditioned media (Fang et al. 2009). In this study, we have demonstrated that ESC-HPMC co-culture apparently promotes the chemotaxis of macrophages more than ESC or HPMC cultured alone, and anti-RANTES-neutralizing antibody can partly inhibit the migration of macrophages in ESCHPMC co-culture. Our previous work has shown that ESC-HPMC co-culture promotes RANTES secretion of ESCs (Yu et al. 2008), which suggests that the retrograded ESCs in peritoneal fluid interact with HPMCs leading to the recruitment of macrophages through increasing the secretion of RANTES. The combination of $\mathrm{E}_{2}$ and TCDD increases the migration of macrophages by stimulating ESC to secret more RANTES and anti-RANTES-neutralizing antibody, and anti-CCR1 or anti-CCR5-neutralizing antibody can decrease but not block completely the migration of macrophages, which suggests that other cytokines and chemokines induced by $\mathrm{E}_{2}$ and TCDD may be involved in this process.
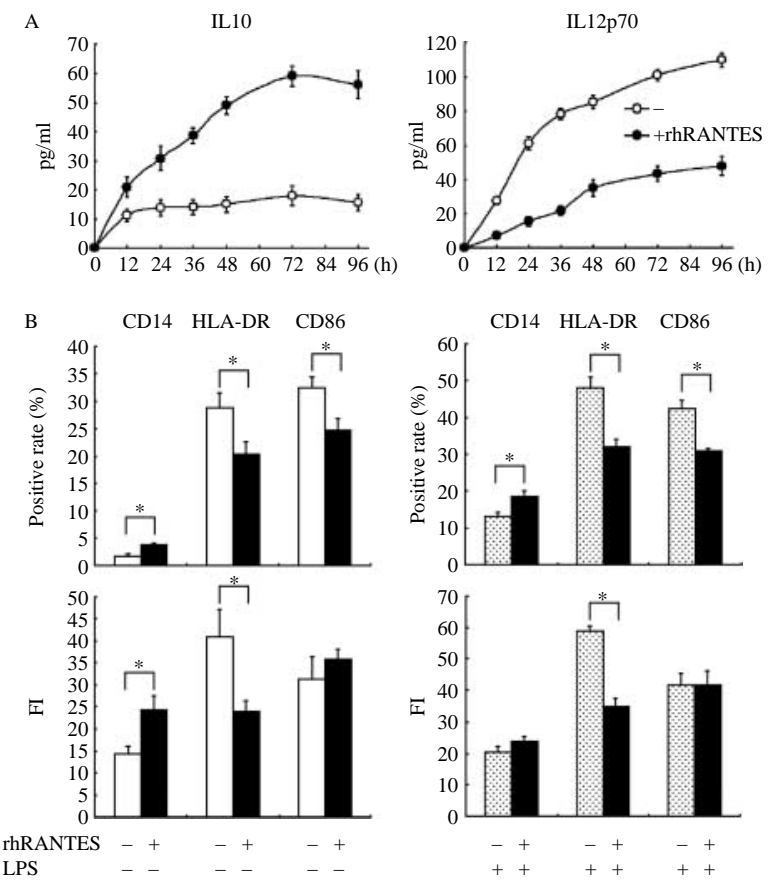

Figure 6 RANTES induces the tolerance phenotype formation of macrophage. U937 cells were cultured for 0-96 h with or without the presence of $10 \mathrm{ng} / \mathrm{ml}$ rhRANTES, and then levels of IL12p70 and IL10 in the supernatant were determined by ELISA (A). U937 cells were treated with or without $10 \mathrm{ng} / \mathrm{ml}$ rhRANTES for $72 \mathrm{~h}$, and were then stimulated with or without LPS $(10 \mathrm{ng} / \mathrm{ml})$ for $24 \mathrm{~h}$. Expressions of CD14, HLA-DR, and CD86 in U937 cells were determined respectively by flow cytometry for positive rate and fluorescence intensity $(\mathrm{FI})$ of the correspondent surface molecules (B). Data are expressed as mean \pm S.E.M. ${ }^{\star} P<0.05$. 


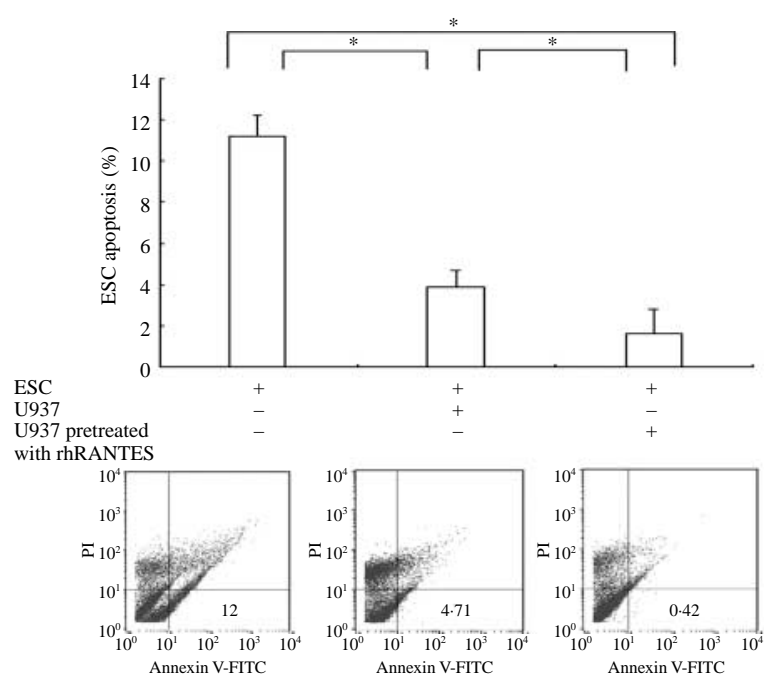

Figure 7 The RANTES-induced tolerant macrophages inhibit apoptosis of ESC. ESCs were cultured alone or co-cultured with U937 cells which were pretreated with or without rhRANTES $(10 \mathrm{ng} / \mathrm{ml})$, and then ESCs were collected to evaluate their apoptosis by flow cytometry. ${ }^{*} P<0.05$.

CCR1 and CCR5 are the receptors of RANTES, and CCR5 is highly expressed in U937 cells, but is not affected by $\mathrm{E}_{2}$ or TCDD (Wang XQ, unpublished observations). In this study, we have found that although CCR1 is lowly expressed in U937 in nature, $\mathrm{E}_{2}$ or TCDD alone can upregulate CCR1 mRNA transcription and protein translation, and the combination of both further enhances this effect. There is a high level of RANTES in peritoneal fluid with endometriosis, which is related to the severity of the disease (Bersinger et al. 2006). Therefore, in the presence of $\mathrm{E}_{2}$ and TCDD, the high expression of CCR5 and CCR1 in macrophages may make them more sensitive to RANTES, thereby promoting their migration to the peritoneal cavity.

After monocytes are recruited into the peritoneal cavity, the abdominal milieu became more complex due to the interaction of ESC, HPMC, and monomacrophages, which aggravates peritoneal inflammation. Hence, we have established different co-culture units of these cells respectively to mimic various peritoneal local inflammation situations of the ectopic milieu. It has been found that all the co-culture units can promote the protein translation of CCR1 in macrophages with comparison to the macrophages cultured alone. Furthermore, the combination of $\mathrm{E}_{2}$ and TCDD can promote CCR1 expression in the macrophages in all the co-culture with ESCs or/and HPMCs. Therefore, the combination of $\mathrm{E}_{2}$ and TCDD can not only promote the secretion of RANTES, but can also upregulate the expression of CCR1 in macrophages. The dual regulation for the chemokine and its receptor determines the pathogenic roles of RANTES in endometriosis.

In this study, we have found that rhRANTES can induce the formation of tolerant macrophages with CD14 ${ }^{\text {high }}$ HLA-DR ${ }^{\text {low }}$ CD $86^{\text {low }}$ phenotype and increased IL10/IL12 ratio. During their exposure to the ectopic milieu, the newly recruited monocytes may be induced tolerant. Our results give a new insight into the formation of tolerant macrophages in endometriosis. RANTES derived from the endometriotic focus-associated cells can change the polarization of macrophage, creating the conditions that redound to endometriosis progression. Therefore, the regulation of the tolerant macrophage phenotype may shed light on developing new therapeutic regimens for endometriosis.

We then address how the tolerant macrophages affect the apoptosis of ESC. It has been found that the apoptosis of ESCs appears significantly decreased when co-cultured with macrophages, which implies that macrophages may inhibit the apoptosis of ESC by secreting soluble cytokines. The macrophages pretreated with RANTES would be tolerant, and these tolerant macrophages show an increased capacity to inhibit the apoptosis of ESC in comparison with the macrophages without RANTES treatment, which indicates that high levels of RANTES produced in the ectopic milieu can induce the formation of tolerant macrophages that in turn promote the ectopic ESC growth in the progression of endometriosis.

In conclusion, based on the results of this study as well as others, a hypothetical model may be proposed to elucidate the onset and progression of endometriosis. The retrograde endometrium activates inflammation, and the interaction of the endometriotic focus-associated cells increases the secretion of RANTES and other cytokines, which is further aggravated by combination of high levels of estrogen with TCDD. The increased RANTES recruits more macrophages into the ectopic milieu. Meanwhile, the interaction of endometriosisassociated cells and the combination of estrogen and TCDD upregulate CCR1 and CCR5 expression in macrophages. Therefore, the macrophages recruited into the ectopic milieu present increased sensitivity to RANTES. The high levels of RANTES not only recruit but also induce macrophages tolerant. These tolerant macrophages inhibit the apoptosis and promote growth of ESC in the endometriotic milieu, which shows potential value for prophylaxis and therapeutics of endometriosis.

\section{Declaration of interest}

The authors declare that there is no conflict of interest that could be perceived as prejudicing the impartiality of the research reported. 


\section{Funding}

This work is supported by National Basic Research Program of China 2006CB944007 (to D-J L), National and Shanghai Leading Academic Discipline Project 211XK22 (to D-J L), Program for Outstanding Medical Academic Leader of Shanghai (to D-J L), and Creative Foundation for Graduate Students of Fudan University EYF157015 (to X-Q W).

\section{Author contribution statement}

$\mathrm{X}-\mathrm{Q} \mathrm{W}$ conducted all the experiments, prepared the figures, and wrote the manuscript. J Y undertook migration, RT-PCR, and western blot analysis, X-Z L completed immunohistochemistry, and Y-L S determined RANTES by ELISA. Y W and L W collected specimens and clinical data. D-J L initiated and supervised the project and edited the manuscript.

\section{References}

Akoum A, Lemay A \& Maheux R 2002 Estradiol and interleukin-1 beta exert a synergistic stimulatory effect on the expression of the chemokine regulated upon activation, normal T cell expressed, and secreted in endometriotic cells. Journal of Clinical Endocrinology and Metabolism 87 5785-5792. (doi:10.1210/jc.2002-020106)

Bersinger NA, von Roten S, Wunder DM, Raio L, Dreher E \& Mueller MD 2006 PAPP-A and osteoprotegerin, together with interleukin-8 and RANTES, are elevated in the peritoneal fluid of women with endometriosis. American Journal of Obstetrics and Gynecology 195 103-108. (doi:10.1016/j.ajog.2005.12.010)

Biswas SK, Gangi L, Paul S, Schioppa T, Saccani A, Sironi M, Bottazzi B, Doni A, Vincenzo B, Pasqualini F et al. 2006 A distinct and unique transcriptional program expressed by tumor-associated macrophages (defective NF-kappa B and enhanced IRF-3/STAT1 activation). Blood 107 2112-2122. (doi:10.1182/ blood-2005-01-0428)

Erwig LP \& Henson PM 2007 Immunological consequences of apoptotic cell phagocytosis. American Journal of Pathology 171 2-8. (doi:10.2353/ajpath.2007.070135)

Fang CL, Han SP, Fu SL, Wang W, Kong N \& Wang XL 2009 Ectopic, autologous eutopic and normal endometrial stromal cells have altered expression and chemotactic activity of RANTES. European Journal of Obstetrics, Gynecology, and Reproductive Biology 143 55-60. (doi:10.1016/j.ejogrb.2008.12.001)

Hasko G, Pacher P, Deitch EA \& Vizi ES 2007 Shaping of monocyte and macrophage function by adenosine receptors. Pharmacology and Therapeutics 113 264-275. (doi:10.1016/j.pharmthera.2006.08.003)

Hill JA, Faris HMP, Schiff I \& Anderson DJ 1988 Characterization of leukocyte subpopulations in the peritoneal fluid of women with endometriosis. Fertility and Sterility 50 216-222.

Hornung D, Ryan IP, Chao VA, Vigne JL, Schriock ED \& Taylor RN 1997 Immunolocalization and regulation of the chemokine RANTES in human endothelial and endometriosis tissues and cells. Journal of Clinical Endocrinology and Metabolism 82 1621-1628. (doi:10.1210/jc.82.5.1621)
Hornung D, Bentzien F, Wallwiener D, Kiesel L \& Taylor RN $2001 a$ Chemokine bioactivity of RANTES in endometriotic and normal endometrial stromal cells and peritoneal fluid. Molecular Human Reproduction 7 163-168. (doi:10.1093/molehr/7.2.163)

Hornung D, Klingel K, Dohrn K, Kandolf R, Wallwiener D \& Taylor RN $2001 b$ Regulated on activation, normal T-cell-expressed and -secreted mRNA expression in normal endometrium and endometriotic implants - assessment of autocrine/paracrine regulation by in situ hybridization. American Journal of Pathology 158 1949-1954.

Kuang DM, Wu Y, Chen N, Cheng JS, Zhuang SM \& Zheng LM 2007 Tumor-derived hyaluronan induces formation of immunosuppressive macrophages through transient early activation of monocytes. Blood 110 587-595. (doi:10.1182/blood-2007-01-068031)

Lebovic DI, Chao VA, Martini JF \& Taylor RN 2001 IL-1beta induction of RANTES (regulated upon activation, normal $\mathrm{T}$ cell expressed and secreted) chemokine gene expression in endometriotic stromal cells depends on a nuclear factor-kappa B site in the proximal promoter. Journal of Clinical Endocrinology and Metabolism 86 4759-4764. (doi:10.1210/jc.86.10.4759)

Martinez FO, Sica A, Mantovani A \& Locati M 2008 Macrophage activation and polarization. Frontiers in Bioscience 13 453-461. (doi:10.2741/2692)

Mosser DM 2003 The many faces of macrophage activation. Journal of Leukocyte Biology 73 209-212. (doi:10.1189/jlb.0602325)

O’Shea JJ \& Murray PJ 2008 Cytokine signaling modules in inflammatory responses. Immunity 28 477-487. (doi:10.1016/ j.immuni.2008.03.002)

Raiter-Tenenbaum A, Baranao RI, Etchepareborda JJ, Meresman GF \& Rumi LS 1998 Functional and phenotypic alterations in peritoneal macrophages from patients with early and advanced endometriosis. Archives of Gynecology and Obstetrics 261 147-157. (doi:10.1007/ s004040050214)

Rier S \& Foster WG 2002 Environmental dioxins and endometriosis. Toxicological Sciences 70 161-170. (doi:10.1093/toxsci/70.2.161)

Shi YL, Luo XZ, Zhu XY, Hua KQ, Zhu Y \& Li DJ 2006 Effects of combined 17 beta-estradiol with TCDD on secretion of chemokine IL-8 and expression of its receptor CXCR1 in endometriotic focusassociated cells in co-culture. Human Reproduction 21 870-879. (doi:10.1093/humrep/dei414)

Shi YL, Luo XZ, Zhu XY \& Li DJ 2007 Combination of 17 beta-estradiol with the environmental pollutant TCDD is involved in pathogenesis of endometriosis via up-regulating the chemokine I-309-CCR8. Fertility and Sterility 88 317-325. (doi:10.1016/ j.fertnstert.2006.11.129)

Wu MH, Sun HS, Lin CC, Hsiao KY, Chuang PC, Pan HA \& Tsai SJ 2002 Distinct mechanisms regulate cyclooxygenase-1 and -2 in peritoneal macrophages of women with and without endometriosis. Molecular Human Reproduction 8 1103-1110. (doi:10.1093/molehr/8.12.1103)

Yu J, Wang Y, Zhou WH, Wang L, He YY \& Li DJ 2008 Combination of estrogen and dioxin is involved in the pathogenesis of endometriosis by promoting chemokine secretion and invasion of endometrial stromal cells. Human Reproduction 23 1614-1626. (doi:10.1093/humrep/den125)

Received in final form 21 June 2010

Accepted 23 August 2010

Made available online as an Accepted Preprint 23 August 2010 